\title{
Wireless Channel Parameters Maximizing TCP Throughput
}

\author{
François Baccelli — Rene L. Cruz — Antonio Nucci
}

$\mathbf{N}^{\circ} \mathbf{5 6 5 2}$

Août 2005

Thème COM 



\title{
Wireless Channel Parameters Maximizing TCP Throughput
}

\author{
François Baccelli *, Rene L. Cruz ${ }^{\dagger}$, Antonio Nucci ${ }^{\ddagger}$ \\ Thème COM — Systèmes communicants \\ Projet TREC
}

Rapport de recherche $\mathrm{n}^{\circ} 5652$ - Août 2005 - 43 pages

\begin{abstract}
We consider a single TCP session traversing a wireless channel, with a constant signal to noise ratio (SINR) at the receiver. We consider the problem of determining the optimal transmission energy per bit, to maximize TCP throughput. Specifically, in the case where direct sequence spread spectrum modulation is used over a fixed bandwidth channel, we find the optimal processing gain $m$ that maximizes TCP throughput. In the case where there is a high signal to noise ratio, we consider the scenario where adaptive modulation is used over a fixed bandwidth channel, and find the optimal symbol alphabet size $M$ to maximize TCP throughput. Block codes applied to each packet for forward error correction can also be used, and in that case we consider the joint optimization of the coding rate to maximize TCP throughput. Finally, we discuss the issue of assigning target SINR values. In order to carry out our analysis, we obtain a TCP throughput formula in terms of the packet transmission error probability $p$ and the transmission capacity $C$, which is of independent interest. In our TCP model, the window size is cut in half for each packet transmission loss, and also cut in half whenever the window size

* INRIA-ENS, francois.baccelli@ens.fr

$\dagger$ UCSD, USA, rcruz@ucsd.edu

$\ddagger$ This author was with Sprint ATL, USA, when this work was initiated. He is now with Narus, USA, anucci@narus. com
\end{abstract}


exceeds the transmission capacity $C$. This formula is then used to characterize the optimal processing gain or the optimal symbol alphabet size as the solution of a simple fixed point equation that depends on the wireless channel parameters and the parameters of the TCP connection.

Key-words: CDMA, adaptive modulation, processing gain, block coding, signal to noise and interference ratio, power control, bandwidth sharing, congestion control, congestion avoidance, additive increase multiplicative decrease algorithm, TCP throughput, optimization, stochastic process, stationary distribution, Mellin transform. 


\section{Paramétres d'un Canal Radio Maximisant le Débit de TCP}

Résumé : Nous étudions dans cet article le débit d'une session TCP sur un canal radio de bande passante donnée et de rapport signal sur bruit et interférence constant au point de réception et nous cherchons à déterminer l'énergie de transmission par bit qui maximise ce débit. Deux scénarios sont étudiés plus spécifiquement: celui d'une modulation à étalement de spectre par séquence directe, pour lequel nous déterminons la valeur optimale du gain de traitement, et celui d'une modulation adaptative, où nous déterminons la taille optimale de l'alphabet. Lorsque les méthodes de codage par blocs de type FEC sont utilisées, nous cherchons à maximiser le débit de la session TCP par une optimisation conjointe du taux de codage et des paramétres des couches physiques évoqués ci-dessus. Nous montrons aussi comment déterminer les rapports signal sur bruit et interférence dans le contexte particulier du CDMA. Notre analyse se fonde sur le calcul explicite de la loi stationnaire du débit d'une session TCP à laquelle est offerte une capacité de transmission de $C$ bits par seconde et qui subit un taux de perte de paquets de $p$. La fenêtre de TCP est divisée par deux soit en cas de perte de paquets soit en cas d'atteinte de la capacité $C$. La formule explicite obtenue pour le débit moyen d'une telle session nous permet de caractériser le gain de traitement ou la taille d'alphabet optimaux comme solutions d'équations de point fixe ne dépendant que des caratéristiques du canal radio et de celles de la session TCP.

Mots-clés : CDMA, modulation adaptative, gain de traitement, codage par bloc, rapport signal sur interférence et bruit, contrôle de puissance, partage de bande passante, contrôle de congestion, évitement de congestion, algorithme des accroissements linéaires et de la décroissance multiplicative, débit de TCP, optimisation, processus aléatoire, distributions stationnaire, transformée de Mellin. 


\section{Introduction}

Cellular wireless networks were originally designed to support voice, which has stringent delay requirements. In these networks, a power control algorithm is used to maintain a target signal to noise ratio (SINR) for each user. The power control algorithm adapts to fast multi-path fading that arises due to mobility of users or the sources of scattering, so that a constant bit rate and a required maximum bit error rate is maintained for each connection, with low transport latency. Thus, for example, when a user encounters a fading channel condition, the transmission power is boosted so that the voice conversation can continue in real time.

These systems have been adapted to carry data as well. A fixed capacity channel may be allocated for a data user. We are generally interested in optimizing the channel parameters in order to provide the best performance for the data user. For simplicity here we assume that a data user corresponds to a single TCP connection. We assume that the channel is allocated a SINR which will be maintained to a constant target value. We focus on the case of a long lived TCP connection. The target SINR value may be adapted over time to respond to user mobility, and we consider the regime where the TCP connection reaches a steady state between updates of the target SINR value.

We consider first the case of CDMA, and consider the problem of optimizing the processing gain $m$ and coding rate $\rho$ to maximize TCP throughput. By adjusting the processing gain and coding rate, we trade-off the bit transmission rate $C$ and packet transmission error rate $p$. By making the processing gain $m$ large we increase the energy per bit and so the packet transmission loss probability $p$ is small. However, the bit transmission rate $C$ is proportional to $1 / m$.

We also consider the context of a high SINR channel over a fixed bandiwidth. In this case, we study adaptive modulation, where the symbol alphabet size $M$ is adapted. In this case the raw transmission rate $C$ is proportional to $\log _{2}(M)$, but the probability of a packet error $p$ grows quickly with $M$. We find the optimal value of the alphabet size $M$ in order to maximize TCP throughput.

To understand the nature of the optimization we consider, it is useful to think of two extremes. At one extreme we can make the bit-transmission rate $C$ 
high, but the packet transmission error rate $p$ will be large. Packet transmission errors will generally cause the TCP protocol to reduce its window size, and in turn decrease throughput. At the other extreme we can make the packet transmission error rate $p$ very small, at the expense of reduced transmission rate $C$. The bit transmission rate $C$ ultimately limits the TCP window size, since buffer overflows will occur when the window gets sufficiently large, and TCP will cut the window size in half, responding to the congestion that occurs at the buffer. Thus, TCP throughput is small at this extreme as well.

In order to find the optimal operating point, we consider a model for analyzing the TCP throughput where packet losses due to transmission errors and packet losses due to congestion events are distinguished. We consider a fluid model where the window size is cut in half for each packet loss due to a transmission error or buffer overflow event. The packet loss probability is $p$, and we assume that a congestion event happens when the window size reaches a value that matches the bit-transmission rate, $C$, on the channel. This model is appropriate for small buffers. This makes sense within e.g. the CDMA context where the bit-transmission rate is small, compared to what is available on wireline networks, and where large downlink buffers would imply large RTTs and hence poor TCP performance as TCP throughput is known to be roughly inversely proportional to RTT.

For this model, we find a formula for the TCP throughput as a function of $p$ and $C$, which is of independent interest. This is a generalization of the well known"square root" formula (e.g. see [12]) for TCP throughput where packet transmission errors and buffer overflows are not separately modeled. We also obtain formulas for the probability density function of the TCP window size.

We find that TCP throughput can be fairly sensitive to the physical layer parameters, i.e. the processing gain $m$ or symbol alphabet size $M$. This suggests it may be advantageous to adapt these parameters according to the environment. In particular, they can be adapted according to the target SINR and the round-trip time (RTT) in order to maximize TCP throughput.

We also discuss the problem of assignment of a target SINR values, and specifically discuss the case of a wireless cellular CDMA downlink.

Before describing our model and analysis in more detail, we first discuss some related work.

$\mathrm{RR} \mathrm{n}^{\circ} 5652$ 


\section{Related Work}

Many authors have considered the general problem of performance of the TCP protocol over wireless links. A comparison of various approaches to the problem is given in [4].

One branch of related work is concerned with the problem of determining whether packet losses are due to congestion or due to transmission errors, so that TCP can go into congestion avoidance mode only when packet losses are due to congestion. A second branch of work considers the approach of splitting the TCP connection at the wire-line/wireless infrastructure boundary, so that the TCP connection is isolated from the packet transmission errors on the wireless channel. A third approach to optimizing TCP performance over wireless channels is to optimize the link layer for TCP performance.

The approach we take in this paper falls into the third category.

Zorzi and Rao [13] considered the effect of correlated errors on TCP throughput. Correlated errors typically occur due to multi-path fading. In our model, we assume a fixed SINR (thanks to power control), so errors can be modeled as independent.

Chaskar, Lakshman, and Madhow [6] consider the use of link layer ARQ over the wireless channel to hide packet transmission errors. In this case, TCP primarily reacts to buffer overflows only. In [6], it is suggested that the physical layer parameters should be optimized so that the buffer overflow probability $q$ times the bandwidth delay product squared is equal to one.

Liu, Goeckel, and Towsley [11] considered the problem of adapting the coding rate $\rho$ to the channel conditions, with the objective of maximizing TCP throughput. It was already noted in [11] that optimal values of operating parameters for the channel for TCP were different than those for UDP.

In [10], Liu, Zhou and Giannakis used simulation to study cross-layer optimization within the adaptive modulation setting.

The main novelty of the present paper is the fact that it provides an analytic framework for this adaptation.

Several recent papers provide analytical formulas for the throughput of a large collection of competing TCP flows with both congestion and transmission error losses (see [2] and the references therein). The main difference with [2] and related papers is that we are here focusing on the rate of a single TCP 
connection constrained to remain below the rate $C$ and subject to both random transmission error losses and to losses that occur when its rate reaches or exceeds $C$.

To the best of our knowledge, this setting, the associated formulas for TCP throughput and the analytic characterizations of the optimal wireless channel parameters that are derived in the present paper are all new.

\section{TCP Throughput}

\subsection{Model}

In this section, we analyze the throughput of a single TCP flow over a wireless channel. Each packet contains $L$ bits, and when it is transmitted over the channel, it is lost with probability $p$. We assume that packet transmission errors are independent. This is reasonable, since we assume that the channel has a fixed signal to interference plus noise ratio $\gamma$. Later on, in Sub-Section 5 , we will consider how the packet transmission loss probability $p$ depends on $\gamma$ and other physical layer parameters. We assume it takes $L / C$ seconds to transmit each packet on the channel. The parameter $C$ is called the raw transmission capacity and has units of bits/sec.

We now consider a fluid model for the TCP flow. Our model is in terms of the parameters $C$ and $p$ discussed above. Consider a random process $X(t)$ that models the instantaneous throughput for the TCP flow. The instantaneous throughput is assumed to be proportional to the TCP window size. Our model for the dynamics of $X(t)$ is as follows. Let $R$ be the round trip delay (assumed constant here), in units of seconds. When there is no loss, at time $t, X(t)$ increases at rate $L / R^{2}$. If $X(t)$ reaches $C$, a congestion event occurs, causing $X(t)$ to be reduced in half to the value $C / 2$. Packet losses are modeled by a time in-homogeneous Poisson process, where the rate of loss at time $t$ is $\lambda(t)=p X(t) / L$. This reflects the fact that the rate of packet loss is higher when the packet rate is higher. When there is a packet loss at time $t$, the value of $X(t)$ is reduced in half.

Of course a more refined model would take into account the presence of a buffer and the fact that losses only take place when this buffer overflows. In

$\mathrm{RR} \mathrm{n}^{\circ} 5652$ 
F. Baccelli, R. Cruz \& A. Nucci

Section 6 , we will show by simulation that the conclusions obtained from our simplified bufferless model are still valid for this refined model.

\subsection{Distributions}

We first define some notation. Let $\alpha=p R^{2} / L^{2}$. Define $\Lambda_{0}=1$ and for $l \geq 1$ define

$$
\Lambda_{l}=\prod_{j=1}^{l}\left(\frac{-4}{2^{2 j}-1}\right) .
$$

For $l \geq 1$ and $n \geq 2$ define

$$
\begin{aligned}
a_{l, n}= & \Lambda_{l-1} \exp \left(-\left(2^{2(l-1)}-1\right) \alpha\left(C / 2^{n}\right)^{2} / 2\right) \\
& {\left[1+\left(\frac{4}{2^{2 l}-1}\right) \exp \left(-(3) 2^{2(l-1)} \alpha\left(C / 2^{n}\right)^{2} / 2\right)\right] . }
\end{aligned}
$$

The following is proved in Appendix 7.2.

Theorem 1 Let $f(x)$ be the stationary probability density function for the instantaneous throughput $X(t)$ of the TCP connection. The density $f$ satisfies the differential equation

$$
\frac{d f(x)}{d x}=\left\{\begin{array}{ll}
-\alpha x f(x) & \text { if } C / 2<x \leq C \\
-\alpha x f(x)+4 \alpha x f(2 x) & \text { if } 0 \leq x<C / 2
\end{array},\right.
$$

where $\alpha=p R^{2} / L^{2}$. The density $f(x)$ is discontinuous at $x=C / 2$ and such that

$$
f\left((C / 2)^{+}\right)-f\left((C / 2)^{-}\right)=f\left(C^{-}\right) .
$$

The density $f(x)$ is given by

$$
f(x)=\sum_{l=0}^{n} V_{n-l} \Lambda_{l} e^{-2^{2 l} \alpha x^{2} / 2}
$$

if $C / 2^{n+1}<x<C / 2^{n}$ for $n \geq 0$, 0 otherwise, where the constants $V_{0}, V_{1}, \ldots$ are the solutions to the equations

$$
V_{1}=V_{0}\left(1+(1 / 3) e^{-3 \alpha C^{2} / 8}\right)
$$




$$
V_{n}=\sum_{l=1}^{n} a_{l, n} V_{n-l} \text { for } n>1
$$

and

$$
\sum_{m=0}^{\infty} V_{m}\left[\int_{C / 2^{m+1}}^{C / 2^{m}} \exp \left(-\alpha x^{2} / 2\right) d x\right]=\left[\sum_{k=0}^{\infty} 2^{-k} \Lambda_{k}\right]^{-1}
$$

It is interesting to note that in all cases examined so far, the constants $V_{n}$ appear to converge to a constant as $n \rightarrow \infty$.

\subsection{Limiting Cases}

First consider the case where $p=\alpha=0$. In this case it can be verified that $\sum_{l=0}^{n} V_{n-l} \Lambda_{l}=0$ for all $n>1$ and hence $f(x)$ is a uniform distribution on $[C / 2, C]$. This is expected since in this case the trajectory of $X(t)$ is a sawtooth pattern varying between $C / 2$ and $C$.

Next consider the case where $C$ approaches infinity. In this case, it can be verified that $V_{n}$ tends to $V_{0}$ for all $n$. Hence in this case we have $f(x)=$ $V_{0}\left(\sum_{l=0}^{\infty} \Lambda_{l} e^{-2^{2 l} \alpha x^{2} / 2}\right)$ for all $x>0$. This is precisely the same result obtained in [3]. This is expected since letting $C$ approach infinity is equivalent to having transmission losses only, which coincides with previous models.

\subsection{Examples}

We now illustrate the above results with some numerical examples. Suppose $C=5, R=100$ and $L=40$. In Figure 1 , the density $f(x)$ of the instantaneous throughout is plotted versus $x$ for two different values of the packet loss probability $p$. The left plot shows the case $p$ small. In this case, note that there is a visible discontinuity in the density at $x=C / 2$, reflecting the jumps from $C$ to $C / 2$ when the instantaneous throughput hits the boundary $C$. The right plot shows the case $p$ large. In this case the packet loss probability is high enough so that it is unlikely that the instantaneous throughput is near the boundary $C$, and thus there is no visible discontinuity in the density at $x=C / 2$. Note that in all cases, $f(x)$ is nearly zero when $x$ is close to zero, which is because packet loss becomes an unlikely event when the instantaneous throughput is near $x=0$.

$\mathrm{RR} \mathrm{n}^{\circ} 5652$ 

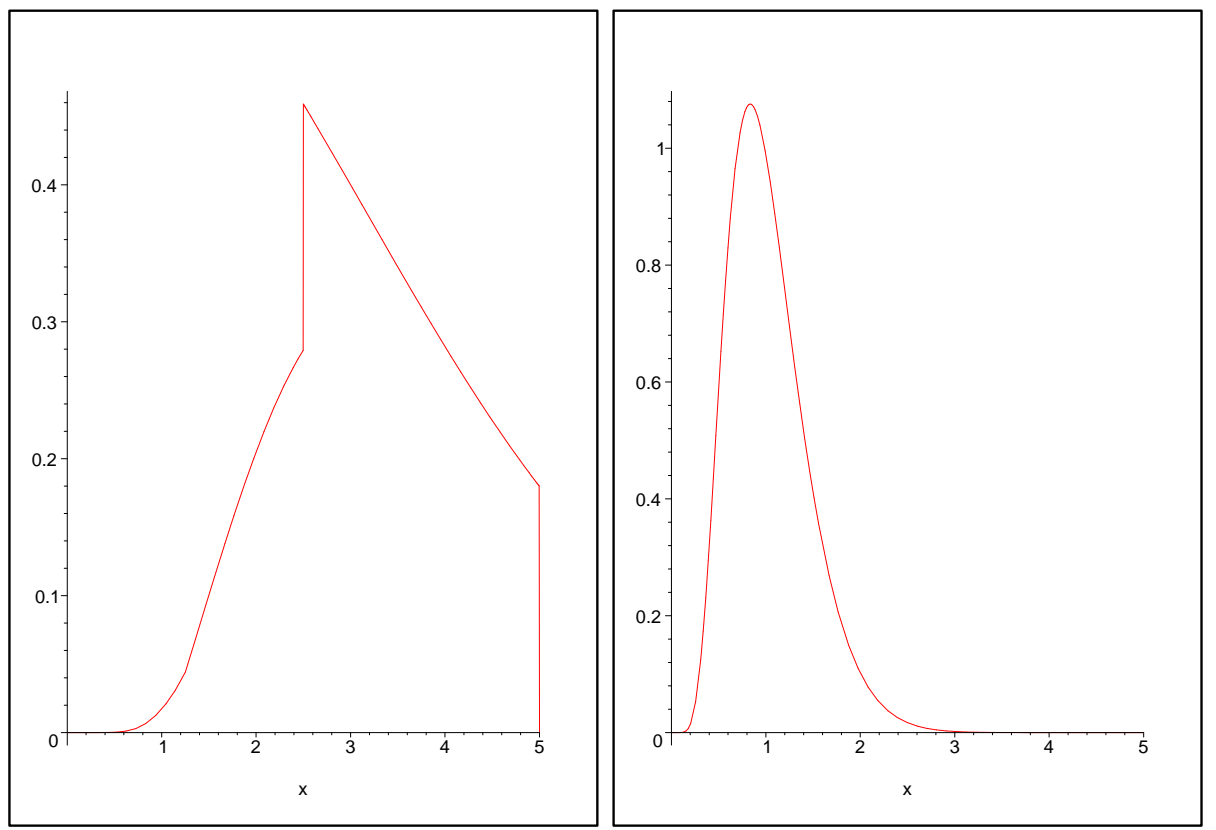

Figure 1: Probability Density of Instantaneous TCP Throughput: Examples

\subsection{Mean Values}

The mean throughput of TCP is given by $T C P(C, p)=\int_{0}^{C} x f(x) d x$. Specifically, by using (5), after some algebraic manipulation, we can show that

$$
T C P(C, p)=\left[\sum_{k=0}^{\infty} 4^{-k} \Lambda_{k}\right] \sum_{m=0}^{\infty} V_{m} \int_{C / 2^{m+1}}^{C / 2^{m}} x \exp \left(-\alpha x^{2} / 2\right) d x .
$$

In the following theorem we obtain a closed form equation for $\operatorname{TCP}(C, p)$, which leads to a simple approximation for $\operatorname{TCP}(C, p)$. Let $\widehat{f}(u)$ be the Mellin transform of $f(x)$ :

$$
\widehat{f}(u)=\int_{0}^{C} f(x) x^{u-1} d x
$$


with $u \geq 1$. Let $\Gamma(u)$ be the Mellin transform of $e^{-x}$, i.e.

$$
\Gamma(u)=\int_{0}^{\infty} e^{-x} x^{u-1} d x .
$$

Note that $\Gamma(\cdot)$ is the well known gamma function and satisfies the identity $\Gamma(u+1)=u \Gamma(u)$. For all $l \geq 0$, define

$$
\Pi_{l}(u)=\prod_{k=0}^{l}\left(1-2^{-u-2 k}\right) .
$$

The following is proved in Appendix 7.3.

Theorem 2 (Mean TCP Throughput) The Mellin transform of the probability density $f(x)$ of the instantaneous TCP throughput is given by

$$
\widehat{f}(u)=\frac{\sum_{l \geq 0} \Pi_{l}(u) C^{u}\left(\frac{\alpha C^{2}}{2}\right)^{l} \frac{\Gamma(u / 2)}{\Gamma(u / 2+l+1)}}{\sum_{l \geq 0} \Pi_{l}(1) C\left(\frac{\alpha C^{2}}{2}\right)^{l} \frac{\Gamma(1 / 2)}{\Gamma(1 / 2+l+1)}} .
$$

In particular, the mean TCP throughput is

$$
T C P(C, p)=\frac{\sum_{l \geq 0} \Pi_{l}(2) C^{2}\left(\frac{\alpha C^{2}}{2}\right)^{l} \frac{1}{(l+1) !}}{\sum_{l \geq 0} \Pi_{l}(1) C\left(\frac{\alpha C^{2}}{2}\right)^{l} \frac{\sqrt{\pi}}{\Gamma(1 / 2+l+1)}} .
$$

Remark 1 We have two different expressions for the mean stationary throughput, obtain by two different methods: that of Equation (9) and that of Theorem 2 above. It would be interesting to prove directly that the two expressions are equal, but we have not been able to do this. We have checked the equality numerically to see if the two expressions yield the same numerical result, and in all cases we tested, the results matched closely.

From (13) we obtain the following approximation, proved in Appendix 7.4.

$\mathrm{RR} \mathrm{n}^{\circ} 5652$ 
Corollary 3 (Approximation to Mean TCP Throughout) The mean TCP throughput satisfies

$$
\begin{aligned}
T C P(C, p) & =\frac{3 C}{4}-\frac{p R^{2} C^{3}}{L^{2}} \frac{11}{256}-\frac{p^{2} R^{4} C^{5}}{L^{4}} \frac{497}{491520} \\
& +\frac{925667}{377487360} \frac{p^{3} R^{6} C^{7}}{L^{6}}+o\left(p^{3} R^{6} C^{7} / L^{6}\right) C .
\end{aligned}
$$

Higher order expansion are easily obtained using e.g. Maple.

As expected, the values of the throughput are insensitive to the value of $C$ if the packet loss probability $p$ is sufficiently high. In fact we have the following corollary. A sketch of the proof is provided in Appendix 7.5.

Corollary 4 For all $p>0$ we have

$$
\lim _{C \rightarrow \infty} T C P(C, p)=\sqrt{\frac{2}{\alpha}} \frac{1}{\sqrt{\pi}} \frac{\Pi_{\infty}(2)}{\Pi_{\infty}(1)}=\frac{1.309}{\sqrt{p} R / L},
$$

The result in Corollary 4 coincides with [3], which is expected since letting $C$ approach $\infty$ is equivalent to considering random transmission losses only.

\section{Optimization of Wireless Channel Parameters}

In this section we consider optimization of the wireless channel parameters to maximize the throughput of the TCP session that passes through it. We can use the formula for the TCP throughput in (14) to evaluate $T C P(C, p)$ as a function of $C$ and $p$. The wireless channel parameters will determine $C$ and $p$, and we wish to maximize $T C P(C, p)$ with respect to these parameters.

We will consider two regimes, the low SINR regime and the high SINR regime. For example, the low SINR regime arises in the context of code-division multiple access (CDMA) systems. In this regime, a processing gain $m$ is used to adjust the transmission data rate, or equivalently the transmission energy used per bit. A large processing gain is generally needed to compensate for low SINR. We shall consider the problem of optimizing the processing gain to maximize TCP throughput. We shall also consider the use of error correction codes, and jointly optimize the coding rate and the processing gain. 
When the SINR is high, it is possible to reliably send more than one bit per transmitted symbol. If the number of possible values of each symbol is $M$, then $M$ is called the alphabet size. The number of bits per symbol is $\log _{2} M$. A large SINR generally enables use of an alphabet size greater than two with low probability of symbol error. We consider the problem of adjusting the alphabet size in order to maximize TCP throughput.

In the next subsection, we present a wireless channel model, which yields formulas for $p$ and $C$ as a function of the wireless channel parameters .

\subsection{Wireless Channel Model}

We first consider a model appropriate for a low SINR at the receiver, in the context of a CDMA network.

\subsubsection{Code Division Multiplexing without FEC}

We will consider the case of code-division multiplexing with direct sequence spread spectrum modulation. In this case, we assume that signals are sent with direct sequence spread spectrum modulation, with a chip duration of $T_{c}$ seconds. Binary Phase Shift Keying (BPSK) is assumed as the underlying modulation scheme. Each bit transmitted is encoded into $m$ chips using a spreading sequence, where $m$ is called the processing gain. The bit transmission rate is thus

$$
C=\frac{1}{m T_{c}}
$$

Typically, many users may transmit at the same time, causing interference at each users receiver. Each user has a receiver which correlates the incoming signal with the spreading sequence used at the transmitter. Let $\gamma$ be the signal to noise plus interference ratio (SINR) at the output of the correlator. We shall define $\gamma$ mathematically when we discuss assignment of SINR values later.

If we model the interference at the correlator output as Gaussian, the probability of a bit error is

$$
B E R=Q(\sqrt{m \gamma})
$$

where $Q(\cdot)$ is the CDF of the zero mean unit variance Gaussian density, i.e. $Q(x)=(1 / \sqrt{2 \pi}) \int_{x}^{\infty} e^{-x^{2} / 2} d x$.

$\mathrm{RR} \mathrm{n}^{\circ} 5652$ 
In the uncoded case that we consider here (no FEC), each packet corresponds to transmission of $L$ bits on the channel. The probability of a packet error is

$$
p=1-(1-Q(\sqrt{m \gamma}))^{L} .
$$

We will use the following approximation for $p$, which is valid when $B E R$ is small:

$$
p \approx L Q(\sqrt{m \gamma})
$$

\subsubsection{Code Division Multiplexing with FEC}

We shall also consider the case where a block code for forward error correction (FEC) is used. In this case, the $L$ bits of each packet are encoded into $N$ bits, where $N \geq L$. The ratio $\rho=L / N$ is called the coding rate. Since we count throughput in terms of information conveyed, the bit transmission capacity in this case is

$$
C=\frac{\rho}{m T_{c}} .
$$

The collection of $N$ bits is called a codeword, and there is a codeword for each of the $2^{L}$ possible bit patterns of a packet. In general, the $N$ codeword bits contain redundancy, so that if bit transmission errors occur, the bit pattern of the originally encoded packet can sometimes be recovered at the receiver. We assume that up to $t$ errors can be corrected, and that $t+1$ or more bit transmission errors result in a packet loss. The probability of a packet error is thus

$$
p=\sum_{j=t+1}^{N}\left(\begin{array}{c}
N \\
j
\end{array}\right)[Q(\sqrt{m \gamma})]^{j}[1-Q(\sqrt{m \gamma})]^{N-j} .
$$

Define $\theta=(t+1) / N$. We use the following large deviations-based estimate for $p$ :

$$
p \approx 2^{N\left[h(\theta)+\theta \log _{2}(q)+(1-\theta) \log _{2}(1-q)\right]},
$$


where $q=Q(\sqrt{m \gamma})$ and $h(x)=-x \log _{2}(x)-(1-x) \log _{2}(1-x)$ is the binary entropy function. Notice that this approximation uses the dominant term of Stirling's formula, and that better expansions could be used.

In order to determine $t$, or equivalently $\theta$, we use the Gilbert-Varshney bound [5], which implies that for a given coding rate $\rho$, there exists a block code with "error correction capability" $\theta$, where $\theta$ satisfies

$$
\rho=1-h(2 \theta) \text {. }
$$

\subsubsection{Adaptive Modulation}

Next, we discuss a scenario appropriate for high SINRs, where more than one bit can be conveyed per transmitted symbol. In the adaptive modulation we consider, the alphabet size $M$ can be varied. Each symbol can thus take on $M$ possible values. The duration of each symbol is $T$ seconds, and the bandwidth of the transmitted signal is proportional to $1 / T$. The bit transmission rate in this scenario is therefore

$$
C=\frac{\log _{2} M}{T} .
$$

We define the signal to noise ratio $\gamma$ in this case to be ratio of the average symbol energy at the receiver to the noise power spectral density. It should be noted that $\gamma$ is also equal to the ratio of the signal power at the receiver to the noise power at the receiver, after channel bandpass filtering.

As stated in [7], for large values of $\gamma$, the probability of a bit error is well approximated by

$$
B E R \sim 2 \frac{\sqrt{M}-1}{\sqrt{M} \log _{2}(M)} Q\left(\sqrt{\frac{3 \gamma}{M-1}}\right) .
$$

Since we have $L$ bits per packet, we use the following approximation for the probability of a packet transmission error, which is valid when $B E R$ is small:

$$
p \sim 2 L \frac{\sqrt{M}-1}{\sqrt{M} \log _{2}(M)} Q\left(\sqrt{\frac{3 \gamma}{M-1}}\right) .
$$

First we consider a single TCP session $i$ which is assigned a given SINR value $\gamma_{i}=\gamma$. We are interested in optimizing the processing gain $m_{i}=m$ and

$\mathrm{RR} \mathrm{n}^{\circ} 5652$ 
the coding rate $\rho_{i}=\rho$ in order to maximize the mean TCP throughput. Recall that we have $C=\frac{\rho}{m T_{c}}$, and that the packet error probability $p$ is a decreasing function of $m$ and an increasing function of $\rho$. We wish to maximize $M=T C P(C, p)$ over all possible values of $m$ and $\rho$.

\subsection{Optimization of TCP Throughput}

In this subsection we consider optimization of TCP throughput in the three cases outlined in the previous subsection.

\subsubsection{Code Division Multiplexing without FEC}

We first present some numerical results. In Figure 4.2.1, we consider the case where $\gamma_{i}=0.03$ and the RTT value is $R=0.1$ second. On the top right curve we plot the TCP throughput as a function of the processing gain $m$. We see that there is fair amount of sensitivity to the processing gain, and there is a unique maximum around $m=450$. To get a better sense of this, on the top left plot we illustrate the TCP throughput ISO curves, i.e. values of $C$ and $p$ that yield the same TCP throughput. Superimposed with these ISO curves, we plot the locus of $(p, C)$ values corresponding to different values of processing gain. We see that the optimum processing gain corresponds to crossing a "knee" of a TCP ISO curve. For reference, we consider optimization of the "UDP throughput" $C(1-p)$ in the bottom two plots in Figure 4.2.1.

In this case we use (16) for $C$ and (19) for $p$ in the expansion for $T C P(C, p)$ in (14), for an explicit optimization of the mean throughput.

Using the approximation $Q(x) \approx \frac{1}{\sqrt{2 \pi} x} e^{-x^{2} / 2}$ in (19), we have

$$
p \sim L e^{-\frac{m \gamma}{2}} \frac{1}{\sqrt{2 \pi m \gamma}} .
$$

If $\alpha C^{2} / 2$ is small, then the first order expansion of the "few losses" case (see (52)), corresponding to the first two terms in (14), gives the following expression for the mean rate

$$
T C P(C, p) \sim \frac{1}{4 T_{c}}\left(\frac{3}{m}-\frac{1}{T_{c}^{2}} \frac{\beta}{m^{3} \sqrt{m}} e^{-\frac{m \gamma}{2}}\right),
$$



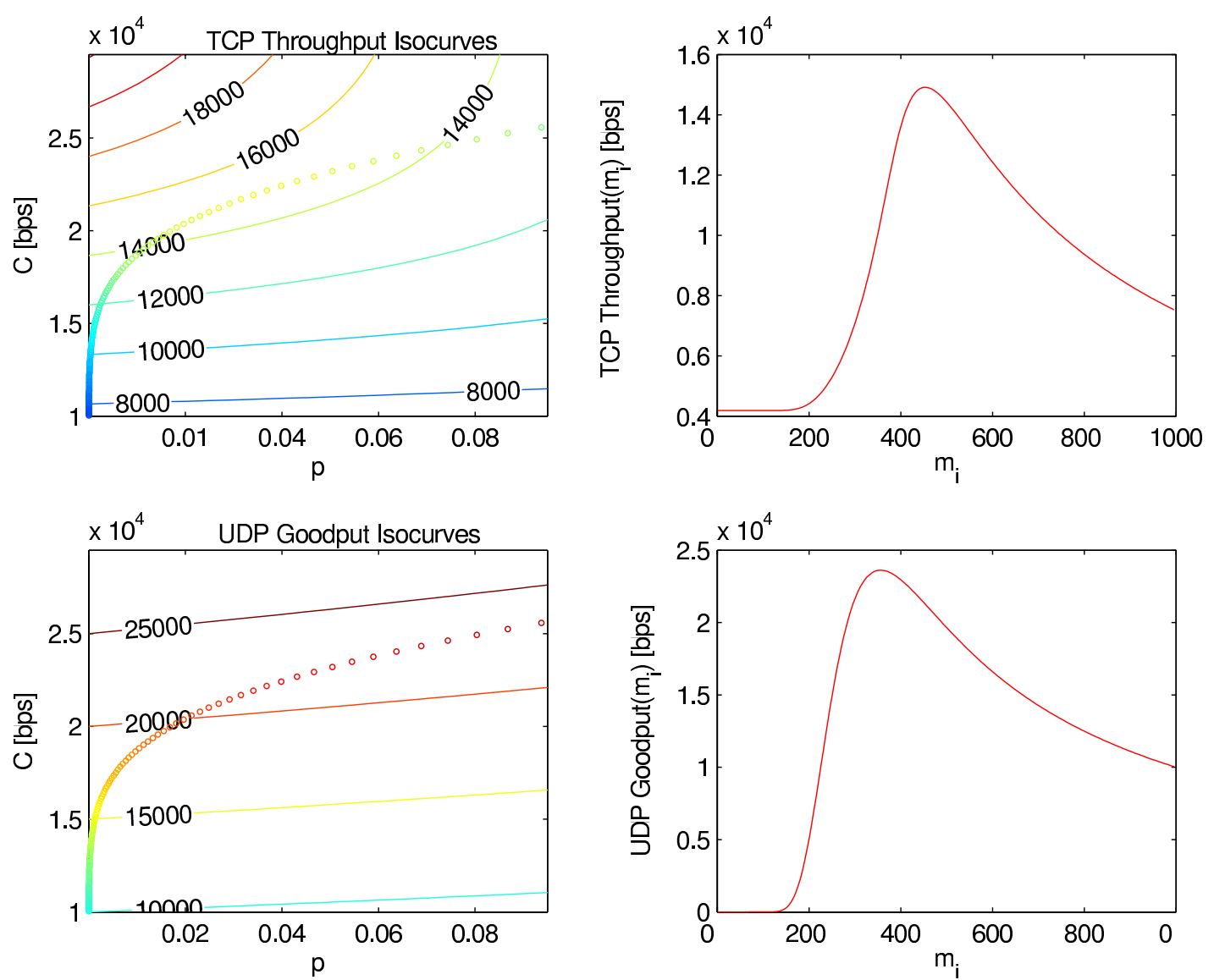

Figure 2: Example: Cross-Layer Optimization Results - Uncoded Case. $\gamma_{i}=$ $0.03, R=0.1$ second

with $\beta=\frac{11 R^{2}}{64 L \sqrt{2 \pi \gamma}}$. Differentiating with respect to $m$, we get that the optimal $m$ solves

$$
\frac{1}{4 T_{c}} \frac{1}{m^{2}}\left(-3+\frac{\beta}{2 T_{c}^{2}}\left(\frac{\gamma}{m \sqrt{m}}+\frac{7}{m^{2} \sqrt{m}}\right) e^{-\frac{m \gamma}{2}}\right)=0,
$$

that is

$$
e^{-\frac{m \gamma}{2}}=\frac{6 T_{c}^{2}}{\beta} \frac{m \sqrt{m}}{\gamma+\frac{7}{m}}
$$

$\mathrm{RR} \mathrm{n}^{\circ} 5652$ 
Higher order approximations of $Q$ can of course be used in the same way when needed. Taking $L=320, R=.1, \gamma=310^{-2}, T_{c}=10^{-7}$, we get $\beta \sim 1.1510^{-6}$; so using the first order approximation for the TCP throughput, the optimal processing gain $m$ satisfies

$$
e^{-.015 m}=4.72710^{-9} \frac{m \sqrt{m}}{.03+\frac{7}{m}} .
$$

The solution is $m=459$. The associated value for $\alpha C^{2} / 2$ is $p C^{2} R^{2} /\left(2 L^{2}\right) \sim$ 0.815 which, upon examination of (14), justifies the use of the few loss approximation (the correction brought by the second order term is appr. 4/1000 of the value given by the first order approximation).

Remark 2 Our model has an interesting relation to [6], where all packet transmission errors are hidden with a link layer mechanism (eg link layer ARQ), so that the only packet losses that TCP reacts to are buffer overflows at the link layer buffer.

Let $W_{b d}$ be the bandwidth delay product (where bandwidth is defined in units of TCP packets/sec). The authors of [6] propose sizing the buffer so that the overflow probability $q$ satisfies $q\left(W_{b d}\right)^{2}=1$. With our method, we find the optimal operating point at $\alpha C^{2} / 2=0.815$, which is equivalent to $p\left(W_{b d}\right)^{2} / 2=$ 0.815 .

\subsubsection{Code Division Multiplexing with FEC}

In this case, we wish to jointly optimize the processing gain $m$ and coding rate $\rho$ in order to maximize TCP throughput. In this case we use (20) for $C$ and (21) for $p$, where $\theta$ is determined from $\rho$ using (22).

In Figure 3, we again consider the case where $\gamma=0.03$ and the RTT value is $R=0.1$ second, and plot results for when both the processing gain $m$ and the coding rate $\rho=L / N$ are jointly optimized. The top left plot shows how the optimum processing gain $m^{*}$ changes as a function of the coding rate $\rho$. On the graph we have also labeled the approximate value of the TCP throughput corresponding to each point. For example, for a coding rate of about 0.4 , the optimum processing gain is about 100, and the corresponding TCP throughput is about $31 \mathrm{Kbps}$. For comparison, in the top right plot we 


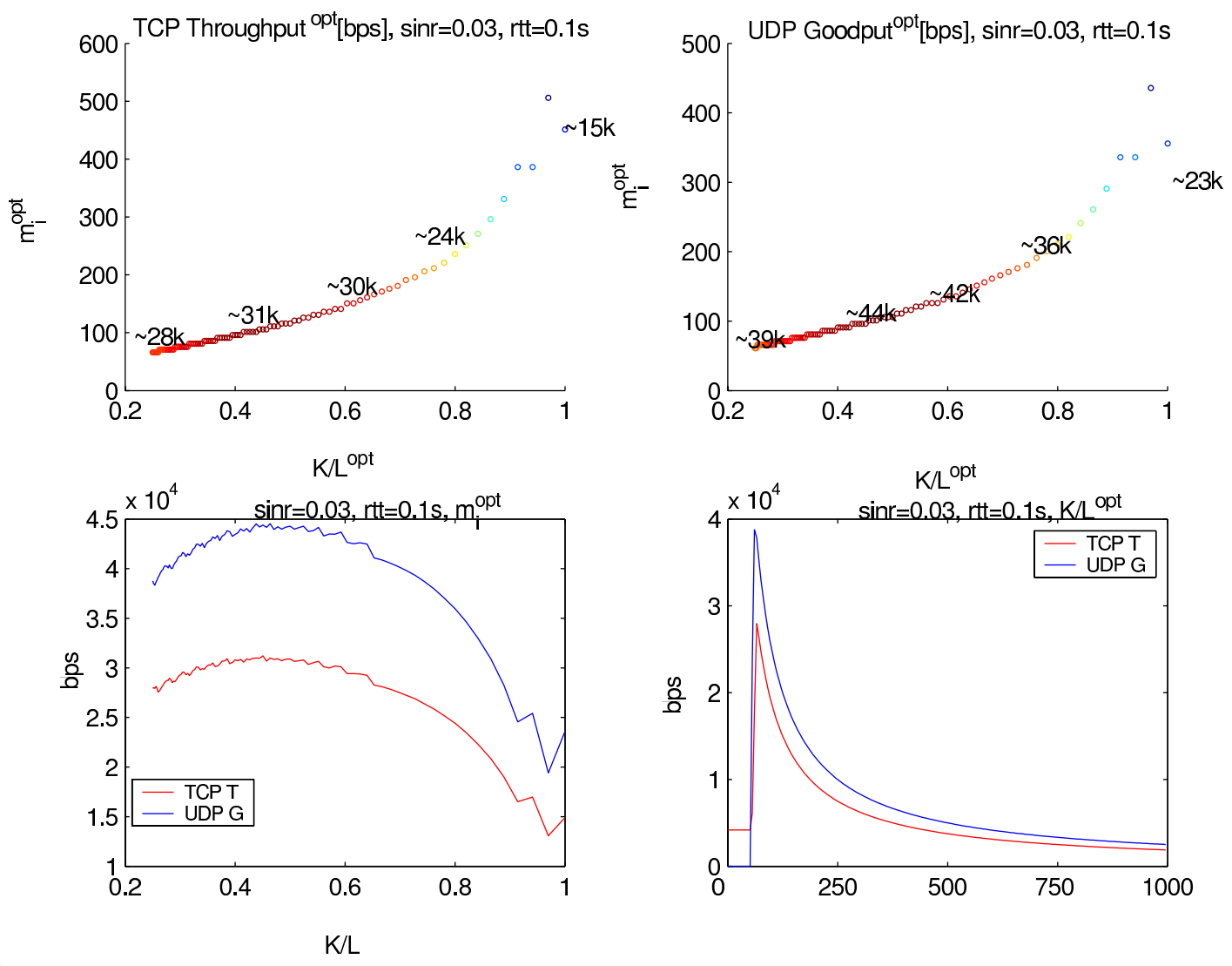

Figure 3: Example: Joint Optimization of Processing Gain $m_{i}$ and Coding Rate $K / L . \gamma_{i}=0.03, R=0.1$ second

show how the optimum processing gain $m^{*}$ changes as a function of the coding rate $\rho$, where we optimize UDP goodput, i.e. $(1-p) C$. In the bottom left plot we show the optimal TCP and UDP throughput as a function of the coding rate $\rho$. In the bottom right plot we show the optimum TCP and UDP throughput as a function of the processing gain $m$.

Next, we describe a procedure for an explicit joint optimization. Define $J=$ $T C P(C, p)$. We are interested in maximizing $J$ with respect to the processing gain $m$ and the coding rate $\rho$. Note that there is a one-to-one relation between

$\mathrm{RR} \mathrm{n}^{\circ} 5652$ 
the coding rate $\rho$ and the parameter $\theta$, through equation (22). For convenience we optimize the TCP throughput $J$ with respect to $m$ and $\theta$, and then the optimal coding rate $\rho^{*}$ is determined by $\theta^{*}$ through $(22)$. Note that $\theta^{*}$ and $m^{*}$ satisfy $\frac{\partial J}{\partial m}=0$ and $\frac{\partial J}{\partial \theta}=0$. Define $F_{1}(m, \theta)=\frac{\partial J}{\partial \theta}$ and $F_{2}(m, \theta)=\frac{\partial J}{\partial m}$. In Appendix 7.6, we provide the calculation of $F_{1}$ and $F_{2}$. We can solve the two non-linear equations $F_{1}(m, \theta)=0$ and $F_{2}(m, \theta)=0$ in order to determine the optimal processing gain $m^{*}$ and the optimal value of the coding rate $\rho^{*}$, where $\rho^{*}$ is found from $\theta^{*}$ by using equation (22).

\subsubsection{Adaptive Modulation}

Using the approximation $Q(x) \approx \frac{1}{\sqrt{2 \pi} x} e^{-x^{2} / 2}$ in (24), we obtain

$$
p \sim 2 L \frac{\sqrt{M}-1}{\sqrt{M} \log _{2}(M)} \frac{\sqrt{(M-1)}}{\sqrt{6 \pi \gamma}} e^{-\frac{3 \gamma}{2(M-1)}} .
$$

Using $C=\log _{2}(M) / T$ and (29) in the expansion (52) gives:

$$
T C P(C, p) \sim \frac{1}{4 T}\left(3 \log _{2}(M)-\frac{\eta}{T^{2}} \frac{(\sqrt{M}-1) \sqrt{M-1}}{\sqrt{M} \log _{2}(M)} e^{-\frac{3 \gamma}{2(M-1)}}\right),
$$

with $\eta=\frac{11 R^{2}}{64 L \sqrt{6 \pi \gamma}}$. Differentiating with respect to $M$, we get that the optimal $M$ solves

$$
\frac{1}{4 T_{c}}\left(\frac{3}{M \log (2)}-\frac{\eta}{T^{2}} e^{-\frac{3 \gamma}{2(M-1)}} k(M)\right)
$$

with

$$
\begin{aligned}
k(M)= & \frac{3 \gamma}{2(M-1)^{2}} \frac{(\sqrt{M}-1)(\sqrt{M-1}}{\sqrt{M} \log _{2}(M)} \\
& +\frac{\log _{2}(M) \frac{2 M-\sqrt{M}-1}{2 \sqrt{M-1}}}{M \log _{2}^{2}(M)} \\
& +\frac{(\sqrt{M}-1) \sqrt{M-1}\left(\frac{\log _{2}(M)}{2 \sqrt{M}}+\frac{1}{\sqrt{M} \log (2)}\right)}{M \log _{2}^{2}(M)} .
\end{aligned}
$$


Hence, the optimal $M$ solves

$$
e^{-\frac{3 \gamma}{2(M-1)}}=\frac{3 T^{2}}{2 \log (2) \eta} \frac{1}{M k(M)} .
$$

Consider the case where $T=10^{-7}, R=.1$, and $L=1500$. In this case, we get $M^{*}=7$ for $\gamma=60$ and $M^{*}=14$ for $\gamma=200$. In Figure 4, we plot the mean TCP throughput as a function of $M$ for $\gamma=60$ and $\gamma=200$. We see that this analysis predicts the optimal value of $M$ closely.
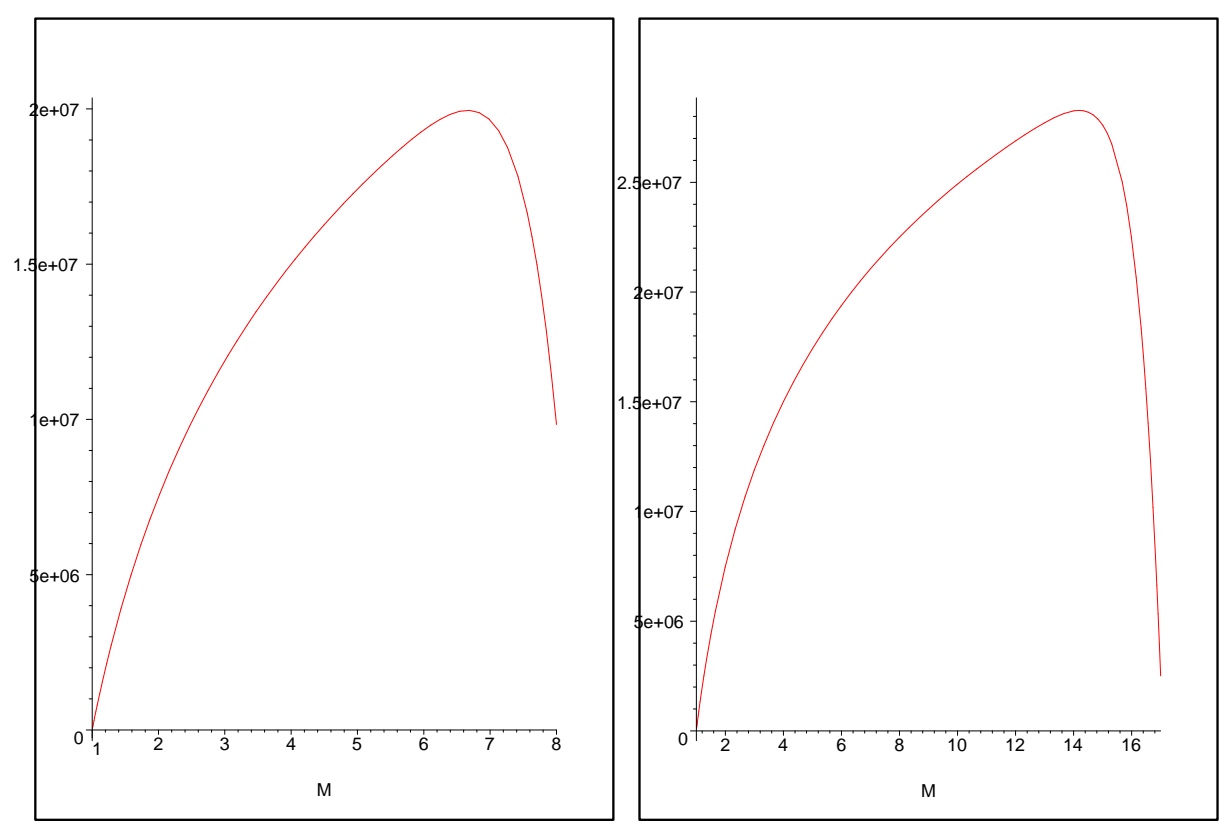

Figure 4: Mean TCP throughput as a function of the constellation size based on $(24)$

\section{$5 \quad$ Assignment of SINR values}

In this section, we discuss the scenario of the downlink of a cellular CDMA network and issues relating to the assignment of SINR values to the different

$\mathrm{RR} \mathrm{n}^{\circ} 5652$ 
users. Suppose there are multiple users on the downlink of a CDMA cellular system, where the users may be associated with different base stations. The signal transmitted for the $i^{\text {th }}$ user at the associated base station is denoted by $s_{i}(t)$ is given by

$$
s_{i}(t)=\sqrt{P_{i}} p_{i}(t) b_{i}(t)
$$

where $p_{i}(t)$ is called the spreading code, $b_{i}(t)$ is called the data signal, and $P_{i}$ is a constant. The spreading code $p_{i}(t)$ takes on values in $\{-1,1\}$ and is constant over intervals of duration $T_{c}$. Specifically,

$$
p_{i}(t)=\sum_{k=-\infty}^{\infty} c_{k}^{i} u\left(t / T_{c}-k\right),
$$

where $u(x)=1$ if $0<x<1$ and $u(x)=0$ otherwise, and for each user $i$, the elements of the sequence $\left\{c_{k}^{i}\right\}_{k=-\infty}^{\infty}$ are either +1 of -1 . The constant $T_{c}$ is called the chip duration and is constant across users.

The data signal $b_{i}(t)$ is also taking its values in $\{-1,1\}$. For the $i^{\text {th }}$ user, we assume that each bit to be sent is repeated $m_{i}$ chips, where $m_{i}$ is an integer. Specifically, we have

$$
b_{i}(t)=\sum_{n=-\infty}^{\infty} b_{n}^{i} u\left(t /\left(m_{i} T_{c}\right)-n\right)
$$

The quantity $m_{i}$ is called the processing gain for user $i$. The data rate for user $i$ is thus $1 /\left(m_{i} T_{c}\right)$. Note that $\left(s_{i}(t)\right)^{2} \equiv P_{i}$, and so the parameter $P_{i}$ is called transmission power for the $i^{\text {th }}$ user.

Typically either pseudo-random or known deterministic sequences are used to define the spreading codes. For purposes of analysis here we assume that $\mathbb{P}\left\{c_{k}^{i}=1\right\}=\mathbb{P}\left\{c_{k}^{i}=-1\right\}=1 / 2$ for all $i, k$, and that $c_{k}^{i}$ is independent of $c_{k^{\prime}}^{i^{\prime}}$ if $i \neq i^{\prime}$ or $k \neq k^{\prime}$. We shall also assume that the data bits are random and independent, i.e. $\mathbb{P}\left\{b_{n}^{i}=1\right\}=\mathbb{P}\left\{b_{n}^{i}=-1\right\}=1 / 2$ for all $i, n$, and that $b_{n}^{i}$ is independent of $b_{n^{\prime}}^{i^{\prime}}$ if $i \neq i^{\prime}$ or $n \neq n^{\prime}$.

We assume a so-called flat fading (i.e. frequency non-selective) channel model. Let $\sqrt{g_{k i}}$ be the signal path gain from the base station associated with user $k$ to the location of user $i$. For example, the useful signal at user $i$ is given by $\sqrt{g_{i i}} s_{i}(t)$. However, the signal intended for another user $k$, namely $\sqrt{g_{k i}} s_{k}(t)$ 
also arrives at the location of user $i$, with possibly a time shift reflecting the different distances between base stations and users. In addition, an external white Gaussian noise signal $n_{i}(t)$ is also present at the receiver for user $i$, with two-sided power spectral density $N_{0}^{i}$. The total signal at the receiver of user $i$ is

$$
r_{i}(t)=\sqrt{g_{i i}} s_{i}(t)+\sum_{k: k \neq i} \sqrt{g_{k i}} s_{k}\left(t-\alpha_{k}^{i}\right)+n_{i}(t) .
$$

The numbers $\alpha_{k}^{i}$ characterize the propagation delays between transmitters and receivers.

Approximating the interference terms as Gaussian, a standard analysis yields that the probability of a bit error, $B E R$, is

$$
B E R=Q\left(\sqrt{m_{i} \Gamma_{i}}\right)
$$

where

$$
\Gamma_{i}=\frac{g_{i i} P_{i}}{(2 / 3)\left(\sum_{k: k \neq i} g_{k i} P_{k}\right)+\left(N_{0}^{i} / T_{c}\right)} .
$$

The quantity $\Gamma_{i}$ is called the signal to interference plus noise ratio, SINR. The quantity $m_{i} \Gamma_{i}$ is known as the " $E b / N o$ ", or energy per bit per noise power density.

Due to mobility of users, the gain values $\left\{g_{k i}\right\}$ change with time. We assume that a closed loop power control algorithm is used, to vary the power values $\left\{P_{i}\right\}$ to maintain the SINR values $\left\{\Gamma_{i}\right\}$ at prescribed values.

Define the SINR vector $\vec{\Gamma}=\left[\Gamma_{1}, \Gamma_{2}, \ldots, \Gamma_{N}\right]^{T}$. We say that an SINR vector $\vec{\gamma}$ is feasible if there exists a set of non-negative power values $\left\{P_{i}\right\}$ such that $\vec{\Gamma}=\vec{\gamma}$. If $\vec{\gamma}$ is feasible, then the power control algorithm sets the transmission powers $\left\{P_{i}\right\}$ accordingly to achieve the target SINR vector $\vec{\gamma}$.

Next we examine the feasibility condition for a target SINR vector. If $\vec{\Gamma} \geq \vec{\gamma}$ then for all $i$ we have

$$
\frac{g_{i i} P_{i}}{(2 / 3)\left(\sum_{k: k \neq i} g_{k i} P_{k}\right)+\sigma_{i}^{2}} \geq \gamma_{i},
$$

where $\sigma_{i}^{2}=N_{0}^{i} / T_{c}$. Equivalently, we have

$$
P_{i}-(2 / 3) \gamma_{i} \sum_{k: k \neq i}\left(g_{k i} / g_{i i}\right) P_{k} \geq \gamma_{i} \sigma_{i}^{2} / g_{i i},
$$

$\mathrm{RR} \mathrm{n}^{\circ} 5652$ 
or in matrix notation, $\vec{P}-F \vec{P} \geq \vec{b}$, where $F=\left\{F_{i, j}\right\}$ is an $N \times N$ matrix with $F_{i i}=0$ and $F_{i, j}=(2 / 3) \gamma_{i}\left(g_{j i} / g_{i i}\right)$ if $i \neq j$, and

$$
\vec{b}=\left[\gamma_{1} \sigma_{1}^{2} / g_{11}, \gamma_{2} \sigma_{2}^{2} / g_{22}, \ldots, \gamma_{N} \sigma_{N}^{2} / g_{N N}\right]^{T}
$$

There exists a non-negative finite $\vec{P}$ satisfying the above, if and only if the spectral radius of the matrix $F$ is less than unity. In this case, the minimal $\vec{P}$ satisfying the above is $\vec{P}^{*}=(I-F)^{-1} \vec{b}$. The minimal power vector $\vec{P}^{*}$ can be found by an iterative distributed algorithm. There may be additional constraints on the power vector, e.g. there is generally a peak power constraint for each base station.

A simple sufficient condition for the spectral radius of $F$ to be less than $\eta$ is that each row sum of $F$ is less than $\eta$. In general, we can set $\eta$ strictly less than 1 as a safety margin, as suggested in [1]. Setting $\eta=1, \vec{\gamma}$ is feasible if for all $i$ we have

$$
(2 / 3) \gamma_{i} \sum_{k: k \neq i}\left(g_{k i} / g_{i i}\right) \leq 1
$$

Note that $g_{k i} / g_{i i}=1$ for all users $k$ that are associated with the same base station that user $i$ is. Furthermore, the value of $g_{k i} / g_{i i}$ is the same for all users $k$ which are associated with the same base station. Suppose there are $B$ base stations. Let $b_{i}$ be the base station associated with user $i$. Assuming $b \neq b_{i}$, define $\alpha_{b}(i)=g_{k i} / g_{i i}$, where $k$ is such that user $k$ is associated with base station $b$. Let $N_{b}$ be the number of users associated with base station $b$. Defining $N_{i}^{i n t}=\sum_{k: k \neq i}\left(g_{k i} / g_{i i}\right)$, we thus have

$$
N_{i}^{i n t}=N_{b_{i}}-1+\sum_{b: b \neq b_{i}} \alpha_{b}(i) N_{b}
$$

We call $N_{i}^{\text {int }}$ the effective number of interfering users for user $i$. The attenuation factor $\alpha_{b}(i)$ can be measured by user $i$ by comparing the power received in pilot tones from base station $b$ and it's assigned base station. The values of $N_{b}$ can be considered to be slowly varying, and reported directly to the base station associated with a given user $i$. If each user $i$ reports the measured value of $\alpha_{b}(i)$ to its associated base station for all $b$, then the value of $N_{i}^{\text {int }}$ is known to the base station associated with user $i, b_{i}$. Hence base station $b_{i}$ can calculate an appropriate value for the target SINR $\gamma_{i}$. In particular, we can 
set $\gamma_{i}=\frac{1.5 \eta}{N_{i}^{i n t}}$, where $\eta$ is a parameter set to a number strictly less than unity, as a safety factor. Note that in general, since the matrix $F$ varies with time, the feasibility of a set of target SINR values also changes with time.

Another simple sufficient condition for the spectral radius of $F$ to be less than $\eta$ is that each column sum is less than $\eta$, i.e. for each $j \in[1, N]$ we have

$$
(2 / 3) \sum_{k: k \neq j} \gamma_{k}\left(g_{j k} / g_{k k}\right) \leq 1
$$

Unlike (34), the condition (35) specifies an explicit coupling between the feasible SINR values of the different users.

In summary, we a set of target SINR values for the users is specified by the vector $\vec{\gamma}$. A target vector $\vec{\gamma}$ is feasible if and only if the spectral radius of $F$ is less than one. Alternatively, we can use either (34) or (35) as the basis for allocating target SINR values $\gamma_{i}$ to the users.

\section{Simulation}

The aim of this section is to analyze the effect of a buffer by simulation. We simulate the case of one base station with a buffer that is shared between $N$ TCP users on the downlink. The general setting is that of $\S 4.1 .1$, with no FEC. The assignment of SINR targets is that of $\S 5$ : all users having the same SINR target since there is only one base station.

We used the hybrid simulator Netscale [8]. More precisely, the simulated dynamics is as follows: as in the mathematical model, each TCP flow is persistent (i.e. always has packets to download); it evolves according to either the slow start or the congestion avoidance phase. In the congestion avoidance phase, it increases its window size $W(t)$ of 1 unit every time $W(t)$ acks and halves it at each packet loss. Losses occur due to either transmission errors (with probability $p$ as defined in (18)) or congestion. The main difference with the dynamics described above is that congestion losses now stem from the interaction of the $N$ flows via the shared buffer. When one or more flows exceed their transmission capacity $C$, as defined in (16), the shared buffer starts filling in and may eventually overflow, creating congestion losses for these flows and possibly for the other flows as well.

$\mathrm{RR} \mathrm{n}^{\circ} 5652$ 
In the simulations, we used a size of $200 \mathrm{~kb}$ for the shared buffer. The minimal value of the RTT is $R=0.5 \mathrm{~s}$. (in the simulation, the RTT increases when the buffer fills in); the packet size is $L=2 \mathrm{~kb}$ and the max window size (not used in the mathematical model but taken into account in the simulation) is of 240 packets.

In all cases (see e.g. Figure 5), we again observe a sharp maximum when plotting the long term average TCP rate of one flow in function of the common value chosen for the processing gains of all flows.

For $N=2$ users, the following table gives the optimal processing gains and the corresponding throughput in kbps as obtained by our mathematical model and by this simulation.

\begin{tabular}{|c||c|c|}
\hline & Optimal processing gain & $\begin{array}{c}\text { Goodput(UDP) } \\
\text { Throughput(TCP) }\end{array}$ \\
\hline UDP & 12 & 128 \\
\hline TCP (theory) & 18 & 355 \\
\hline TCP (simulation) & 15 & 431 \\
\hline
\end{tabular}

Figure 5 plots the UDP and TCP goodput in function of the processing gain in the case with $N=10$ users.

We observe that the optimal processing gain is smaller than what is predicted by theory in the case with 2 users, whereas it is slightly larger for 10 users.

However our simulations suggest that the presence of a shared buffer does not modify our global conclusions that the maximum TCP throughput is achieved by a precise tuning of the processing gain which is quite different from that for a UDP flow.

\section{Appendix}

\subsection{Variance of Interference}

To calculate the variance of the interference, it suffices to show that $\rho_{i, k}$ has variance $(2 / 3) m_{i}\left(T_{c}\right)^{2}$. Note that $\rho_{i, k}$ is a zero mean random variable. Since 


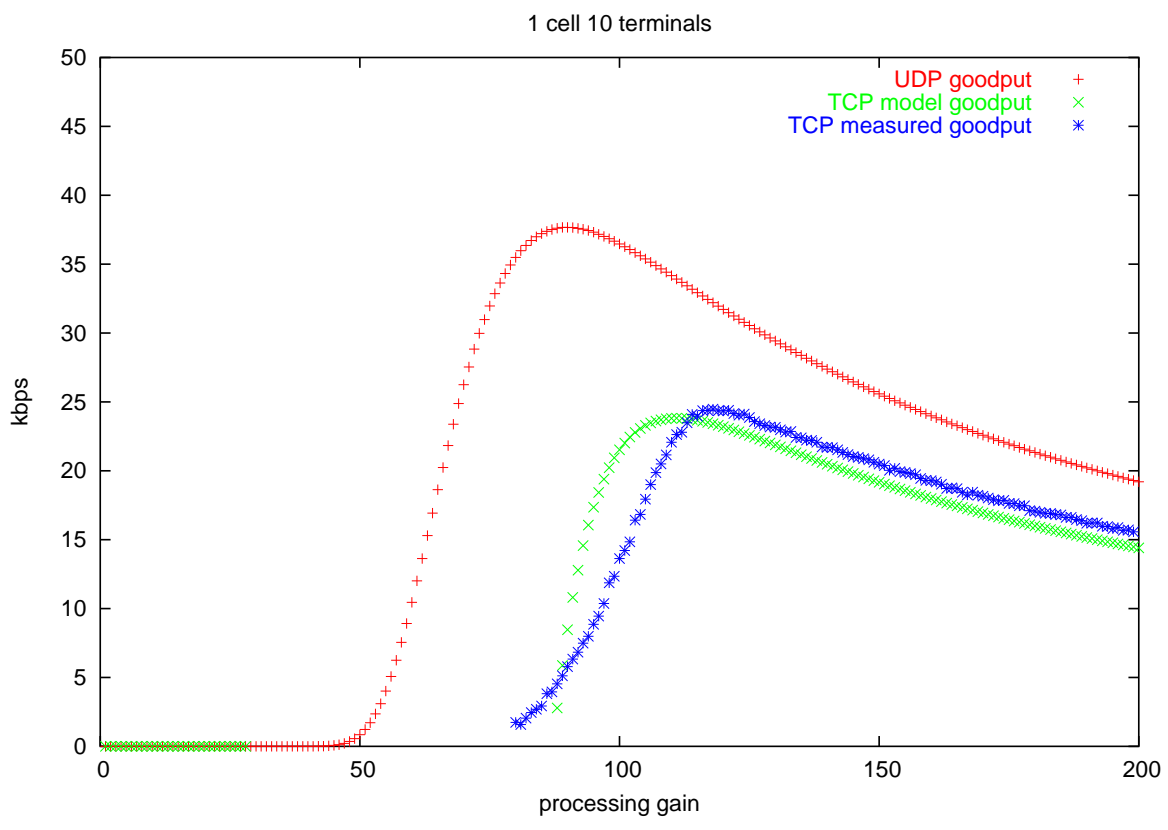

Figure 5: $N=10$ : TCP and UDP goodput as a function of the processing gain by Netscale simulation (in blue) and according to our mathematical model (in green).

the statistics of $b_{k}\left(t-\alpha_{k}^{i}\right) p_{k}\left(t-\alpha_{k}^{i}\right)$ are identical to that of $p_{k}\left(t-\alpha_{k}^{i}\right)$, we have

$\mathrm{RR} \mathrm{n}^{\circ} 5652$ 
that $E\left[\left(\rho_{i, k}\right)^{2}\right]$ is

$$
\begin{aligned}
E\left[\left(\int_{0}^{m_{i} T_{c}}{ }_{i}(t) p_{k}\left(t-\alpha_{k}^{i}\right) b_{k}\left(t-\alpha_{k}^{i}\right) d t\right)^{2}\right] & \\
= & E\left[\left(\int_{0}^{m_{i} T_{c}} p_{i}(t) p_{k}\left(t-\alpha_{k}^{i}\right) d t\right)^{2}\right] \\
= & E\left[\left(\sum_{j=1}^{m_{i}} \int_{(j-1) T_{c}}^{j T_{c}} p_{i}(t) p_{k}\left(t-\alpha_{k}^{i}\right) d t\right)^{2}\right] \\
= & E\left[\left(\sum_{j=1}^{m_{i}} c_{j}^{i} \int_{(j-1) T_{c}}^{j T_{c}} p_{k}\left(t-\alpha_{k}^{i}\right) d t\right)^{2}\right] \\
= & E\left[\sum_{j=1}^{m_{i}} \sum_{j^{\prime}=1}^{m_{i}} c_{j}^{i} c_{j^{\prime}}^{i} \int_{(j-1) T_{c}}^{j T_{c}} p_{k}\left(t-\alpha_{k}^{i}\right) d t \int_{\left(j^{\prime}-1\right) T_{c}}^{j T_{c}} p_{k}\left(t-\alpha_{k}^{i}\right) d t\right] \\
= & \sum_{j=1}^{m_{i}} \sum_{j^{\prime}=1}^{m_{i}} E\left[c_{j}^{i} c_{j^{\prime}}^{i}\right] E\left[\int_{(j-1) T_{c}}^{j T_{c}} p_{k}\left(t-\alpha_{k}^{i}\right) d t \int_{\left(j^{\prime}-1\right) T_{c}}^{j T_{c}} p_{k}\left(t-\alpha_{k}^{i}\right) d t\right] \\
= & \sum_{j=1}^{m_{i}} E\left[\left(c_{j}^{i}\right)^{2}\right] E\left[\left(\int_{(j-1) T_{c}}^{j T_{c}} p_{k}\left(t-\alpha_{k}^{i}\right) d t\right)^{2}\right] \\
= & \sum_{j=1}^{m_{i}} E\left[\left(\int_{(j-1) T_{c}}^{j T_{c}} p_{k}\left(t-\alpha_{k}^{i}\right) d t\right)^{2}\right] .
\end{aligned}
$$


Hence

$$
\begin{aligned}
E\left[\left(\int_{0}^{m_{i} T_{c}} p_{i}(t) p_{k}\left(t-\alpha_{k}^{i}\right) b_{k}\left(t-\alpha_{k}^{i}\right) d t\right)^{2}\right] \\
=m_{i} E\left[\left(\int_{0}^{T_{c}} p_{k}\left(t-\alpha_{k}^{i}\right) d t\right)^{2}\right] \\
=m_{i} E\left[\left(\int_{0}^{T_{c}} p_{k}(t-\alpha) d t\right)^{2}\right] \\
=m_{i} E\left[\left(\alpha c_{-1}^{k}+\left(T_{c}-\alpha\right) c_{0}^{k}\right)^{2}\right] \\
=m_{i}\left(E\left[\alpha^{2}\right]+E\left[\left(T_{c}-\alpha\right)^{2}\right]\right) \\
=2 m_{i} E\left[\alpha^{2}\right] \\
=2 m_{i}\left(1 / T_{c}\right) \int_{0}^{T_{c}} x^{2} d x \\
=(2 / 3) m_{i} T_{c}^{2} .
\end{aligned}
$$

Therefore, the variance of the interference is

$$
\sum_{k: k \neq i}(2 / 3) g_{k i} m_{i}\left(T_{c}\right)^{2} P_{k}
$$

\subsection{Proof of Theorem 1 (Density of Instantaneous TCP Throughput)}

Let $f_{t}(x)$ be the probability density function for $X(t)$. We wish to examine how the density $f_{t}$ evolves over time, and obtain the steady state density if it exists.

For $x<C / 2$ we have that $f_{t}(x) d x$ is

$$
\begin{aligned}
& \mathbb{P}\{X(t) \in[x, x+d x]\} \\
= & \mathbb{P}\left\{X(t-d t) \in\left[x-\left(L / R^{2}\right) d t, x-\left(L / R^{2}\right) d t+d x\right]\right\}(1-(p / L) x d t) \\
& +\mathbb{P}\{X(t-d t) \in[2 x, 2 x+2 d x]\}(p / L) 2 x d t \\
= & f_{t-d t}\left(x-\left(L / R^{2}\right) d t\right)(1-(p / L) x d t) d x+f_{t-d t}(2 x)(p / L) 2 x 2 d x d t .
\end{aligned}
$$

Rewriting this, we have

$$
f_{t}(x)=f_{t-d t}\left(x-\left(L / R^{2}\right) d t\right)(1-(p / L) x d t)+f_{t-d t}(2 x)(p / L) 4 x d t
$$

$\mathrm{RR} \mathrm{n}^{\circ} 5652$ 
or

$$
\begin{aligned}
f_{t}(x)-f_{t-d t}\left(x-\left(L / R^{2}\right) d t\right)= & -f_{t-d t}\left(x-\left(L / R^{2}\right) d t\right)(p / L) x d t \\
& +f_{t-d t}(2 x)(p / L) 4 x d t .
\end{aligned}
$$

Thus,

$$
\begin{aligned}
& \frac{f_{t}(x)-f_{t-d t}(x)+f_{t-d t}(x)-f_{t-d t}\left(x-\left(L / R^{2}\right) d t\right)}{d t} \\
=- & f_{t-d t}\left(x-\left(L / R^{2}\right) d t\right)(p / L) x+f_{t-d t}(2 x)(p / L) 4 x .
\end{aligned}
$$

Letting $d t$ and $d x$ approach zero, we obtain the following equation (à la FokkerPlanck)

$$
\frac{\partial f_{t}(x)}{\partial t}+\left(L / R^{2}\right) \frac{\partial f_{t}(x)}{\partial x}=-(p / L) x f_{t}(x)+4(p / L) x f_{t}(2 x) .
$$

Similarly, for $x>C / 2$ we have that $f_{t}(x) d x$ is

$$
\begin{aligned}
\mathbb{P}\{X(t) \in[x, x+d x]\}= & \mathbb{P}\left\{X(t-d t) \in\left[x-\left(L / R^{2}\right) d t, x-\left(L / R^{2}\right) d t+d x\right]\right\} \\
& (1-(p / L) x d t) \\
= & f_{t-d t}\left(x-\left(L / R^{2}\right) d t\right)(1-(p / L) x d t) d x .
\end{aligned}
$$

Rewriting this, we have

$$
f_{t}(x)=f_{t-d t}\left(x-\left(L / R^{2}\right) d t\right)(1-(p / L) x d t)
$$

or

$$
f_{t}(x)-f_{t-d t}\left(x-\left(L / R^{2}\right) d t\right)=-f_{t-d t}\left(x-\left(L / R^{2}\right) d t\right)(p / L) x .
$$

Thus,

$$
\begin{aligned}
& \frac{f_{t}(x)-f_{t-d t}(x)+f_{t-d t}(x)-f_{t-d t}\left(x-\left(L / R^{2}\right) d t\right)}{d t} \\
& =-f_{t-d t}\left(x-\left(L / R^{2}\right) d t\right)(p / L) x .
\end{aligned}
$$


Letting $d t$ and $d x$ approach zero, we obtain

$$
\frac{\partial f_{t}(x)}{\partial t}+\left(L / R^{2}\right) \frac{\partial f_{t}(x)}{\partial x}=-(p / L) x f_{t}(x) .
$$

We assume that $f_{t}(x) \rightarrow f(x)$ as $t \rightarrow \infty$, i.e. that $f$ is the steady state density of $X(t)$. From the above, setting $\frac{\partial f_{t}(x)}{\partial t}=0$, it follows that $f$ satisfies the differential equation

$$
\frac{d f(x)}{d x}=\left\{\begin{array}{ll}
-\alpha x f(x) & \text { if } C / 2<x \leq C \\
-\alpha x f(x)+4 \alpha x f(2 x) & \text { if } 0 \leq x<C / 2
\end{array},\right.
$$

where $\alpha=p R^{2} / L^{2}$, which proves (3).

For $C / 2<x<C$, note that a solution to the equation $\frac{d f(x)}{d x}=-\alpha x f(x)$ is given by

$$
f(x)=V_{0} \exp \left(-\alpha x^{2} / 2\right) \text { for } x \in(C / 2, C),
$$

where $V_{0}$ is any constant. It is interesting to note that $f$ has a Gaussian shape. For $(C / 4)<x<C / 2$, we therefore have

$$
\frac{d f(x)}{d x}=-\alpha x f(x)+4 V_{0} \alpha x \exp \left(-4 \alpha x^{2} / 2\right) .
$$

The solution to this is

$$
f(x)=V_{1} \exp \left(-\alpha x^{2} / 2\right)+V_{0}(-4 / 3) \exp \left(-4 \alpha x^{2} / 2\right)
$$

for $x \in(C / 4, C / 2)$, where $V_{1}$ is any constant. For $x \in(C / 8, C / 4), f(x)$ must therefore satisfy

$$
\begin{aligned}
\frac{d f(x)}{d x}= & -\alpha x f(x)+V_{1} 4 \alpha x \exp \left(-4 \alpha x^{2} / 2\right) \\
& +V_{0}(-4 / 3) 4 x \alpha \exp \left(-16 \alpha x^{2} / 2\right)
\end{aligned}
$$

A solution to this is

$$
\begin{aligned}
f(x)= & V_{2} \exp \left(-\alpha x^{2} / 2\right)+V_{1}(-4 / 3) \exp \left(-4 \alpha x^{2} / 2\right) \\
& +V_{0}(-4 / 3)(-4 / 15) \exp \left(-16 \alpha x^{2} / 2\right),
\end{aligned}
$$

RR $n^{\circ} 5652$ 
for $x \in(C / 8, C / 4)$, where $V_{2}$ is any constant. The general solution can be found by induction to be

$$
f(x)=V_{n} \exp \left(-\alpha x^{2} / 2\right)+\sum_{l=1}^{n} V_{n-l}\left[\prod_{j=1}^{l}\left(\frac{-4}{2^{2 j}-1}\right)\right] \exp \left(-2^{2 l} \alpha x^{2} / 2\right)
$$

for $x \in\left(C / 2^{n+1}, C / 2^{n}\right)$, which proves (5). Next we find the value of the constants $V_{0}, V_{1}, V_{2}, \ldots$ Note that $f_{t}(C / 2+d x) d x$ is

$$
\begin{aligned}
& \mathbb{P}\{X(t) \in[C / 2, C / 2+d x]\} \\
&=\mathbb{P}\left\{X(t-d t) \in\left[C / 2-\frac{L}{R^{2}} d t, C / 2-\frac{L}{R^{2}} d t+d x\right]\right\} \\
& \quad(1-(p / L) C / 2 d t) \\
&+\mathbb{P}\left\{X(t-d t) \in\left[C-\frac{L}{R^{2}} d t, C-\frac{L}{R^{2}} d t+d x\right]\right\} \\
& \quad(1-(p / L) C d t) \\
&=f_{t-d t}\left(C / 2-\frac{L}{R^{2}} d t\right)(1-(p / L) C / 2 d t) d x \\
&+f_{t-d t}\left(C-\frac{L}{R^{2}} d t\right)(1-(p / L) C d t) d x .
\end{aligned}
$$

Letting $d x$ and $d t$ approach zero, and $t \rightarrow \infty$, we obtain

$$
f\left((C / 2)^{+}\right)=f\left((C / 2)^{-}\right)+f\left(C^{-}\right),
$$

which proves (4). This can be used to find a relationship between $V_{0}$ and $V_{1}$ : using (39), we have

$$
\begin{aligned}
V_{0} \exp \left(-\alpha C^{2} / 8\right)= & V_{1} \exp \left(-\alpha C^{2} / 8\right)+V_{0}(-4 / 3) \exp \left(-\alpha C^{2} / 2\right) \\
& +V_{0} \exp \left(-\alpha C^{2} / 2\right)
\end{aligned}
$$

Thus,

$$
V_{0}=V_{1}+V_{0}(-4 / 3) \exp \left(-3 \alpha C^{2} / 8\right)+V_{0} \exp \left(-3 \alpha C^{2} / 8\right),
$$

or equivalently $V_{1}=V_{0}\left(1+(1 / 3) \exp \left(-3 \alpha C^{2} / 8\right)\right)$, which is equation (6). 
For $n>1, f_{t}\left(C / 2^{n}+d x\right) d x$ is

$$
\begin{aligned}
& \mathbb{P}\left\{X(t) \in\left[\frac{C}{2^{n}}, \frac{C}{2^{n}}+d x\right]\right\} \\
&=\mathbb{P}\left\{X(t-d t) \in\left[\frac{C}{2^{n}}-\frac{L}{R^{2}} d t, \frac{C}{2^{n}}-\frac{L}{R^{2}} d t+d x\right]\right\}\left(1-(p / L) C / 2^{n} d t\right) \\
&+ \mathbb{P}\left\{X(t-d t) \in\left[\frac{C}{2^{n-1}}-2 d x, \frac{C}{2^{n-1}}\right]\right\}(p / L) \frac{C}{2^{n-1}} d t \\
&= f_{t-d t}\left(\frac{C}{2^{n}}-\frac{L}{R^{2}} d t\right)\left(1-(p / L) \frac{C}{2^{n}} d t\right) d x+f_{t-d t}\left(\frac{C}{2^{n-1}}-\frac{L}{R^{2}} d t\right)(p / L) \frac{C}{2^{n-1}} d t .
\end{aligned}
$$

Letting $d t$ approach zero, and $t \rightarrow \infty$, we obtain $f\left(\left(C / 2^{n}\right)^{+}\right)=f\left(\left(C / 2^{n}\right)^{-}\right)$. Thus we have

$$
\begin{aligned}
& V_{n} \exp \left(-\alpha\left(\frac{C}{2^{n}}\right)^{2} / 2\right)+\sum_{l=1}^{n} V_{n-l}\left[\prod_{j=1}^{l}\left(\frac{-4}{2^{2 j}-1}\right)\right] \exp \left(-2^{2 l} \alpha\left(\frac{C}{2^{n}}\right)^{2} / 2\right) \\
& =V_{n-1} \exp \left(-\alpha\left(\frac{C}{2^{n}}\right)^{2} / 2\right) \sum_{l=1}^{n-1} V_{n-1-l}+\left[\prod_{j=1}^{l}\left(\frac{-4}{2^{2 j}-1}\right)\right] \exp \left(-2^{2 l} \alpha\left(\frac{C}{2^{n}}\right)^{2} / 2\right) .
\end{aligned}
$$

Therefore we have that $V_{n} \exp \left(-\alpha\left(\frac{C}{2^{n}}\right)^{2} / 2\right)$ is equal to

$$
\begin{aligned}
& V_{n-1}\left[\exp \left(-\alpha\left(\frac{C}{2^{n}}\right)^{2} / 2\right)+(4 / 3) \exp \left(-4 \alpha\left(\frac{C}{2^{n}}\right)^{2} / 2\right)\right] \\
& -\sum_{l=2}^{n} V_{n-l}\left[\prod_{j=1}^{l}\left(\frac{-4}{2^{2 j}-1}\right)\right] \exp \left(-2^{2 l} \alpha\left(\frac{C}{2^{n}}\right)^{2} / 2\right) \\
& +\sum_{l=1}^{n-1} V_{n-1-l}\left[\prod_{j=1}^{l}\left(\frac{-4}{2^{2 j}-1}\right)\right] \exp \left(-2^{2 l} \alpha\left(\frac{C}{2^{n}}\right)^{2} / 2\right) \\
& =V_{n-1}\left[\exp \left(-\alpha\left(\frac{C}{2^{n}}\right)^{2} / 2\right)+(4 / 3) \exp \left(-4 \alpha\left(\frac{C}{2^{n}}\right)^{2} / 2\right)\right] \\
& -\sum_{l=2}^{n} V_{n-l}\left[\prod_{j=1}^{l}\left(\frac{-4}{2^{2 j}-1}\right)\right] \exp \left(-2^{2 l} \alpha\left(\frac{C}{2^{n}}\right)^{2} / 2\right) \\
& +\sum_{l=2}^{n} V_{n-l}\left[\prod_{j=1}^{l-1}\left(\frac{-4}{2^{2 j}-1}\right)\right] \exp \left(-2^{2(l-1)} \alpha\left(\frac{C}{2^{n}}\right)^{2} / 2\right)
\end{aligned}
$$

$\mathrm{RR} \mathrm{n}^{\circ} 5652$ 
which is equal to

$$
\begin{array}{r}
V_{n-1}\left[\exp \left(-\alpha\left(\frac{C}{2^{n}}\right)^{2} / 2\right)+(4 / 3) \exp \left(-4 \alpha\left(\frac{C}{2^{n}}\right)^{2} / 2\right)\right] \\
+\sum_{l=2}^{n} V_{n-l}\left[\prod_{j=1}^{l-1}\left(\frac{-4}{2^{2 j}-1}\right)\right] \exp \left(-2^{2(l-1)} \alpha\left(\frac{C}{2^{n}}\right)^{2} / 2\right) \\
{\left[1+\left(\frac{4}{2^{2 l}-1}\right) \exp \left(-(3) 2^{2(l-1)} \alpha\left(\frac{C}{2^{n}}\right)^{2} / 2\right)\right] .}
\end{array}
$$

Thus we have

$$
\begin{gathered}
V_{n}=\sum_{l=1}^{n} V_{n-l}\left[\prod_{j=1}^{l-1}\left(\frac{-4}{2^{2 j}-1}\right)\right] \exp \left(-\left(2^{2(l-1)}-1\right) \alpha\left(C / 2^{n}\right)^{2} / 2\right) \\
{\left[1+\left(\frac{4}{2^{2 l}-1}\right) \exp \left(-(3) 2^{2(l-1)} \alpha\left(C / 2^{n}\right)^{2} / 2\right)\right],}
\end{gathered}
$$

which is equation (7).

To find the constants $\left\{V_{n}\right\}$ we will also need the following equation:

$$
\begin{aligned}
1 & =\int_{0}^{C} f(x) d x \\
& =\int_{C / 2}^{C} f(x) d x+\sum_{n=1}^{\infty} \int_{C / 2^{n+1}}^{C / 2^{n}} f(x) d x \\
& =V_{0} \int_{C / 2}^{C} \exp \left(-\alpha x^{2} / 2\right) d x+\sum_{n=1}^{\infty} \int_{C / 2^{n+1}}^{C / 2^{n}} \\
& {\left[V_{n} \exp \left(-\alpha x^{2} / 2\right)+\sum_{l=1}^{n} V_{n-l}\left[\prod_{j=1}^{l}\left(\frac{-4}{2^{2 j}-1}\right)\right]\right.} \\
& \sum_{m=0}^{\infty} V_{m}\left[\int_{C / 2^{m+1}}^{C / 2^{m}} \exp \left(-\alpha x^{2} / 2\right) d x\right] \\
& {\left[1+\sum_{k=1}^{\infty} 2^{-k} \prod_{j=1}^{k}\left(\frac{-4}{2^{2 j}-1}\right)\right] }
\end{aligned}
$$


which proves (8).

The proof of (9) is along the same lines.

\subsection{Proof of Theorem 2 (Mean TCP Throughput)}

We have

$$
\frac{d f(x)}{d x}=\left\{\begin{array}{ll}
-\alpha x f(x) & \text { if } C / 2<x \leq C \\
-\alpha x f(x)+4 \alpha x f(2 x) & \text { if } 0 \leq x<C / 2
\end{array},\right.
$$

where $\alpha=p R^{2} / L^{2}$. The function $f$ is differentiable everywhere but at $C / 2$ where it is differentiable on the right and on the left and with a jump such that:

$$
f\left((C / 2)^{+}\right)=f\left((C / 2)^{-}\right)+f\left(C^{-}\right) .
$$

Note that $f(x)=0$ for $x \notin[0, C]$. Let $\widehat{f}(u)$ be the Mellin transform of $f(x)$ :

$$
\widehat{f}(u)=\int_{0}^{\infty} f(x) x^{u-1} d x
$$

with $u \geq 1$. Multiplying both sides of equation (40) by $x^{u}$ and integrating w.r.t. $x$, we get

$$
f\left(C^{-}\right) C^{u}-f\left((C / 2)^{+}\right)(C / 2)^{u}-u \int_{C / 2}^{C} f(x) x^{u-1} d x=-\alpha \int_{C / 2}^{C} f(x) x^{u+1} d x(
$$

and

$$
\begin{gathered}
f\left((C / 2)^{-}\right)(C / 2)^{u}-u \int_{0}^{C / 2} f(x) x^{u-1} d x \\
=-\alpha \int_{0}^{C / 2} f(x) x^{u+1} d x+\alpha \frac{1}{2^{u}} \int_{0}^{C} f(v) v^{u+1} d v .
\end{gathered}
$$

Adding (43) and (44) and using (41), we obtain

$$
u \widehat{f}(u)=\left(1-\frac{1}{2^{u}}\right)\left(C^{u} f\left(C^{-}\right)+\alpha \widehat{f}(u+2)\right) .
$$

$\mathrm{RR} \mathrm{n}^{\circ} 5652$ 
Note the similarity of equation (45) to the well known equation $\Gamma(u+1)=$ $u \Gamma(u)$, satisfied by the Gamma function. Motivated by this observation, we now express the unknown function $\widehat{f}(\cdot)$ in terms of a new unknown function $g(\cdot)$ by defining

$$
\widehat{f}(u)=g(u) \Gamma(u / 2)\left(\frac{2}{\alpha}\right)^{u / 2}
$$

Multiplying (45) by $\left(\frac{\alpha}{2}\right)^{u / 2} \frac{1}{2} \frac{1}{\Gamma(u / 2+1)}$, one gets

$$
g(u)=\left(1-\frac{1}{2^{u}}\right)(\Psi(u)+g(u+2)),
$$

with

$$
\Psi(u)=\frac{f\left(C^{-}\right)}{2} C^{u}\left(\frac{\alpha}{2}\right)^{u / 2} \frac{1}{\Gamma(u / 2+1)} .
$$

For all integers $l$ let

$$
\Pi_{l}(u)=\prod_{k=0}^{l}\left(1-2^{-u-2 k}\right) .
$$

Note that the infinite product $\Pi_{\infty}(u)$ is well defined and non zero. The general solution of (47) is

$$
g(u)=\sum_{l \geq 0} \Pi_{l}(u) \Psi(u+2 l)+\Pi_{\infty}(u) h(u),
$$

with $h(u)$ some finite periodic function with period 2 . Hence

$$
\widehat{f}(u)=\frac{f\left(C^{-}\right)}{2} \sum_{l \geq 0} \Pi_{l}(u) C^{u+2 l}\left(\frac{\alpha}{2}\right)^{l} \frac{\Gamma(u / 2)}{\Gamma(u / 2+l+1)}+\Pi_{\infty}(u) h(u) \Gamma(u / 2)\left(\frac{2}{\alpha}\right)^{u / 2} .
$$

Since the density $f(x)$ has its support on $[0, C]$, the function $\widehat{f}(u)$ grows at most $C^{u}$ when $u$ tends to $\infty$. The growth of the function

$$
\frac{f\left(C^{-}\right)}{2} \sum_{l \geq 0} \Pi_{l}(u) C^{u+2 l}\left(\frac{\alpha}{2}\right)^{l} \frac{\Gamma(u / 2)}{\Gamma(u / 2+l+1)}
$$


is easily seen to be at most $C^{u}$. So if $h($.$) is non zero, the order of \widehat{f}(u)$ would be that of

$$
\Gamma(u / 2)\left(\frac{2}{\alpha}\right)^{u / 2}
$$

which is a contradiction with the fact that the order of this function is at most $C^{u}$ when $u$ tends to $\infty$. Hence $h(u) \equiv 0$ necessarily, so that

$$
\widehat{f}(u)=\frac{f\left(C^{-}\right)}{2} \sum_{l \geq 0} \Pi_{l}(u) C^{u+2 l}\left(\frac{\alpha}{2}\right)^{l} \frac{\Gamma(u / 2)}{\Gamma(u / 2+l+1)} .
$$

The constant $f\left(C^{-}\right)$is determined by the relation

$$
1=\widehat{f}(1)=\frac{f\left(C^{-}\right)}{2} \sum_{l \geq 0} \Pi_{l}(1) C^{1+2 l}\left(\frac{\alpha}{2}\right)^{l} \frac{\Gamma(1 / 2)}{\Gamma(1 / 2+l+1)} .
$$

We finally get the following expression for the Mellin transform of interest:

$$
\widehat{f}(u)=\frac{\sum_{l \geq 0} \Pi_{l}(u) C^{u+2 l}\left(\frac{\alpha}{2}\right)^{l} \frac{\Gamma(u / 2)}{\Gamma(u / 2+l+1)}}{\sum_{l \geq 0} \Pi_{l}(1) C^{1+2 l}\left(\frac{\alpha}{2}\right)^{l} \frac{\Gamma(1 / 2)}{\Gamma(1 / 2+l+1)}},
$$

which proves (12). The mean TCP throughput is $T C P(C, p)=\widehat{f}(2)$, which proves (13).

\subsection{Proof of Corollary 3 (Approximation to Mean TCP Throughput)}

We have

$$
\begin{aligned}
\operatorname{TCP}(C, p) & =\widehat{f}(2) \\
& =\frac{3 C}{4}\left[\frac{1+\frac{15}{64} p R^{2} C^{2} / L^{2}+o\left(p R^{2} C^{2} / L^{2}\right)}{1+\frac{28}{96} p R^{2} C^{2} / L^{2}+o\left(p R^{2} C^{2} / L^{2}\right)}\right] \\
& =\frac{3 C}{4}-\frac{p R^{2} C^{3}}{L^{2}} \frac{11}{256}+o\left(p R^{2} C^{2} / L^{2}\right) C .
\end{aligned}
$$

$\mathrm{RR} \mathrm{n}^{\circ} 5652$ 
Using the Maple software tool, we obtain the following second order expansion

$$
\widehat{f}(2)=\frac{3 C}{4}-\frac{p R^{2} C^{3}}{L^{2}} \frac{11}{256}-\frac{p^{2} R^{4} C^{5}}{L^{4}} \frac{497}{491520}+o\left(p^{2} R^{4} C^{4} / L^{4}\right) C .
$$

This suggests using the approximation for the "few losses" case:

$$
\widehat{f}(2) \approx \frac{3 C}{4}-\frac{p R^{2} C^{3}}{L^{2}} \frac{11}{256} .
$$

\subsection{Proof of Corollary 4 (sketch)}

From Stirling's formula, if $n$ is large,

$$
\frac{A^{n}}{n !} \sim\left(\frac{A e}{n}\right)^{n} \frac{1}{\sqrt{2 \pi n}} .
$$

Hence

$$
\log \left(\frac{A^{n}}{n !}\right) \sim n \log (A)+n-n \log (n)-\frac{1}{2} \log (n)-\frac{1}{2} \log (2 \pi) .
$$

For $A$ large, the last expression is maximized with respect to $n$ when $n$ satisfies

$$
\log (A)-\log (n)-\frac{1}{2 n} \approx 0,
$$

i.e., for $n$ close to $A$.

If $\alpha C^{2}=p R^{2} C^{2} / L^{2}$ is large, the last observation shows that the dominant term in the numerator of (13) is approximately

$$
\frac{2}{\alpha} \Pi_{\infty}(2)\left(\frac{\alpha C^{2}}{2}\right)^{\frac{\alpha C^{2}}{2}},
$$

whereas the dominant term in the denominator is

$$
\sqrt{\frac{2}{\alpha}} \Pi_{\infty}(1) \sqrt{\pi}\left(\frac{\alpha C^{2}}{2}\right)^{\frac{\alpha C^{2}}{2}} .
$$

Hence the ratio tends to

$$
\widehat{f}(2)=\sqrt{\frac{2}{\alpha}} \frac{1}{\sqrt{\pi}} \frac{\Pi_{\infty}(2)}{\Pi_{\infty}(1)}=\frac{1.309}{\sqrt{p} R / L} .
$$




\subsection{Calculation of $\frac{\partial J}{\partial \theta}$ and $\frac{\partial J}{\partial m}$.}

We have

$$
\frac{\partial J}{\partial \theta}=\frac{\partial J}{\partial C} \frac{\partial C}{\partial \theta}+\frac{\partial J}{\partial p} \frac{\partial p}{\partial \theta}
$$

From (14), we have

$$
\frac{\partial J}{\partial p} \approx-\frac{R^{2} C^{3}}{L^{2}} \frac{11}{256}
$$

and

$$
\frac{\partial J}{\partial C} \approx \frac{3}{4}-p \frac{R^{2} C^{2}}{L^{2}} \frac{33}{256}
$$

Now

$$
\begin{aligned}
\frac{\partial p}{\partial \theta} & =\frac{\partial}{\partial \theta}\left[2^{N\left[h(\theta)+\theta \log _{2}(q)+(1-\theta) \log _{2}(1-q)\right]}\right] \\
& =p(L / \rho) \log _{2}\left(\frac{q(1-\theta)}{\theta(1-q)}\right)
\end{aligned}
$$

Next, we have

$$
\begin{aligned}
\frac{\partial C}{\partial \theta} & =\frac{\partial}{\partial \theta}\left(\frac{\rho}{m T_{c}}\right) \\
& =\left(\frac{2}{m T_{c}}\right) \log _{2}\left(\frac{2 \theta}{1-2 \theta}\right)
\end{aligned}
$$

We thus have an equation expressing $\frac{\partial J}{\partial \theta}$ in terms of $m$ and $\theta$, say

$$
\frac{\partial J}{\partial \theta}=F_{1}(m, \theta)
$$

where the function $F_{1}(m, \theta)$ is determined by substitution in the equations above.

Next, we have

$$
\frac{\partial J}{\partial m}=\frac{\partial J}{\partial C} \frac{\partial C}{\partial m}+\frac{\partial J}{\partial p} \frac{\partial p}{\partial m}
$$

$\mathrm{RR} \mathrm{n}^{\circ} 5652$ 
The quantities $\frac{\partial J}{\partial C}$ and $\frac{\partial J}{\partial p}$ were given above. We have

$$
\frac{\partial C}{\partial m}=-\frac{\rho}{m^{2} T_{c}}
$$

In order to compute $\frac{\partial p}{\partial m}$ note that

$$
\frac{\partial p}{\partial m}=\frac{\partial p}{\partial q} \frac{\partial q}{\partial m}
$$

We have

$$
\begin{aligned}
\frac{\partial p}{\partial q} & \approx \frac{\partial}{\partial q}\left[2^{N\left[h(\theta)+\theta \log _{2}(q)+(1-\theta) \log _{2}(1-q)\right]}\right] \\
& =p L(1 /(\rho \log 2))(\theta / q-(1-\theta) /(1-q)) \\
& =p L(1 /(\rho \log 2))\left(\frac{\theta-q}{q(1-q)}\right)
\end{aligned}
$$

and

$$
\frac{\partial q}{\partial m}=-\frac{1}{\sqrt{2 \pi}} e^{-m \gamma / 2} \sqrt{\frac{\gamma}{4 m}} .
$$

We thus have an equation expressing $\frac{\partial J}{\partial m}$ in terms of $m$ and $\theta$, say

$$
\frac{\partial J}{\partial m}=F_{2}(m, \theta)
$$

where the function $F_{2}(m, \theta)$ is determined by substitution in the equations above.

\section{Conclusion}

In this paper we have considered the optimization of wireless channel parameters in order to optimize the throughput of a single TCP connection passing through the channel. Effectively, we consider optimization of the transmission energy consumed per bit. We assumed that the signal to noise ratio at the receiver of the channel is held constant. We considered two regimes - the first 
where the SINR value is low and where energy per bit is increased by increasing the processing gain, and the second where the SINR value is high and the energy per bit can be decreased by increasing the symbol alphabet size. In both case, we found that the TCP throughput can be fairly sensitive to these parameters, and our results suggest that these parameters should be set carefully and as a function of the system scenario. For example, when the round trip time (RTT) is large, the impact of a packet loss is higher, so the energy per bit should generally increase with increasing RTT. We have also found that the use of forward error correction, when the coding rate is optimized, can significantly increase TCP throughput.

In a network context, the assignment of SINR values can be a very complex problem, particularly when the path gains are changing quickly with time. The results of this paper can perhaps be used as a foundation for considering various approaches for assignment of SINR values, where the interaction between users is explicitly taken into account.

One topic for further investigation is the relation of our results to [6]. In particular, it would be interesting to look at the average rate of congestion events and compare this with the rate of packet transmission loss events, assuming that the energy per bit has been optimized. It would also be interesting to evaluate the total rate at which the window size gets halved at the optimal operating point, and find a simple relationship between this rate and the other parameters of the model.

\section{Acknowledgments}

We would like to thank J. Bolot for his inputs to this line of thoughts as well as D. Hong and L. Fournié for their comments on the paper and their help on the simulations used in $\S 6$. We are also grateful to G. Giannakis for having suggested the idea to apply our ideas, which were initially motivated by CDMA, to adaptive modulation.

$\mathrm{RR} \mathrm{n}^{\circ} 5652$ 
F. Baccelli, R. Cruz \& A. Nucci

\section{References}

[1] F. Baccelli, B. Blaszczyszyn and M. Karray, "Up and Downlink Admission and Congestion Control and Maximal Load in Large Homogeneous CDMA Networks" ACM MONET, vol. 9, no. 6, Dec. 2004.

[2] F. Baccelli, K.B. Kim and D. De Vleeschauwer, "Analysis of the Competition between Wired, DSL and Wireless Users in an Access Network", Proceedings of IEEE INFOCOM'05, March 2005.

[3] F. Baccelli, D. R. McDonald, and J. Reynier, "A Mean-Field Model for Multiple TCP Connections through a Buffer Implementing RED," Performance Evaluation, vol. 49, pp. 77-97, 2002.

[4] H. Balakrishnan, V. N. Padmanabhan, S. Seshan and R. H. Katz. "A comparison of mechanisms for improving TCP performance over wireless links," IEEE/ACM Trans. on Networking, 5(6):756-769, 1997

[5] R.E. Blahut, Theory and Practice of Error-Control Codes, AddisonWesley, Reading, Massachusetts, 1983.

[6] H. Chaskar, T. V. Lakshman and U. Madhow, "TCP Over Wireless with Link Level Error Control: Analysis and Design Methodology", IEEE/ACM Trans. Networking, vol. 7, no. 5, pp. 605-615, October 1999 http://www.ece.ucsb.edu/Faculty/Madhow/publications.html

[7] A. Goldsmith, Wireless Communications, Cambridge University Press, 2005 .

[8] D. Hong, http://www.n2nsoft.com/, 2005.

[9] T. V. Lakshman and U. Madhow, "The performance of TCP/IP for networks with high bandwidth-delay products and random loss," IEEE/ACM Trans. Networking, vol. 5, no. 3, pp. 336-350, June 1997. http://www.ece.ucsb.edu/Faculty/Madhow/publications.html

[10] Q. Liu, S. Zhou and G. Giannakis, " Queuing with Adaptive Modulation and Coding over Wireless Links: Cross-Layer Analysis and Design", IEEE Tr. on Wireless Communications, Vol. 4, No. 3, May 2005. 
[11] B. Liu, D. L. Goeckel and D. Towsley, "TCP-cognizant adaptive forward error correction in wireless networks," GLOBECOM 2002 - IEEE Global Telecommunications Conference, vol. 21, no. 1, November 2002 pp. 21392144 http://www.cs.uml.edu/ bliu/pub/wirelessTCP.pdf

[12] M. Mathis, J. Semke, J. Mahdavi, and T. Ott, "The macroscopic behaviour of the TCP congestion avoidance algorithm," ACM Computer Comm. Review, vol. 27, no. 3, pp. 67-82, July 1997.

[13] M. Zorzi and R. R. Rao. "The effect of correlated errors on the performance of TCP," IEEE Comm. Letter, 1(5):127-129, 1997

$\mathrm{RR} \mathrm{n}^{\circ} 5652$ 


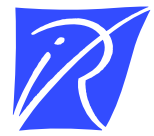

Unité de recherche INRIA Rocquencourt

Domaine de Voluceau - Rocquencourt - BP 105 - 78153 Le Chesnay Cedex (France)

Unité de recherche INRIA Futurs : Parc Club Orsay Université - ZAC des Vignes

4, rue Jacques Monod - 91893 ORSAY Cedex (France)

Unité de recherche INRIA Lorraine : LORIA, Technopôle de Nancy-Brabois - Campus scientifique

615, rue du Jardin Botanique - BP 101 - 54602 Villers-lès-Nancy Cedex (France)

Unité de recherche INRIA Rennes : IRISA, Campus universitaire de Beaulieu - 35042 Rennes Cedex (France)

Unité de recherche INRIA Rhône-Alpes : 655, avenue de l'Europe - 38334 Montbonnot Saint-Ismier (France)

Unité de recherche INRIA Sophia Antipolis : 2004, route des Lucioles - BP 93 - 06902 Sophia Antipolis Cedex (France)

INRIA - Domaine de Voluceau - Rocquencourt, BP 105 - 78153 Le Chesnay Cedex (France)

http://www.inria.fr

ISSN 0249-6399 